\title{
Las salvaguardias y la sostenibilidad del sector automotriz de la provincia de Tungurahua - año 2014 a junio del 2017.
}

\section{The safeguards and sustainability of the automotive sector in the province of Tungurahua - year 2014 to June 2017.}

Paola Katherine Díaz Sánchez. ${ }^{1}$, Mónica Patricia Mayorga Díaz. ${ }^{2}$, Ana Alexandra López Jara. ${ }^{3}$ María Teresa Espinosa Jaramillo. ${ }^{4}$

\section{https://doi.org/10.33262/visionariodigital.v4i2.1216}

\begin{abstract}
.
Safeguards are emergency measures adopted by countries that due to their imports have caused damage to the national product or an imbalance in the balance of payments, this has been a subject of debate in Ecuador, especially in recent fiscal years, due to the implementation of 2,963 subheadings with surcharges of $45 \%, 25 \%, 15 \%$ and $5 \%$ for capital goods, raw materials and consumer goods. This decision was caused by the appreciation of the dollar and the fall in oil prices, affecting multiple sectors, the automotive sector being one of them, in reference to its economic impact, for the country and its sustainability implementation to help the automotive sector, in Ecuador is divided into four groups, which are: 1. various economic, 2. environmental impacts, 3. impact on resources or materials, and 4. social impacts, is one that allows companies to deliver their products or services without compromising future resources or skills of future generations .; In this sense, this research analyzes the impact that safeguards have caused on the economic situation of the automotive

${ }^{1}$ Profesional independiente, Ambato, Ecuador, kataangi@ hotmail.com

${ }^{2}$ Universidad Regional Autónoma de Los Andes, Ambato, Ecuador, monikmayorgad@gmail.com , ORCID:0000-

${ }^{3}$ Universidad Católica de Cuenca, Cuenca, Ecuador, annylopezjara@hotmail.com

${ }^{4}$ Universidad de Zulia, Zulia Venezuela, espinosa.auditores@gmail.com
\end{abstract} 0003-3071-236X 
sector, through the documentary, financial and economic analysis of fiscal years 2014 until the first half of 2017.

Keywords: Safeguards, sustainability, automotive sector, profitability, economic situation.

\section{Resumen.}

Las salvaguardias constituyen medidas de urgencia adoptadas por países que por sus importaciones han causado daño al producto nacional o un desequilibrio en la balanza de pagos, esto ha sido tema de debate en el Ecuador, en especial en los últimos ejercicios fiscales, debido a la implementación de 2.963 subpartidas con sobretasas del $45 \%, 25 \%, 15 \%$ y $5 \%$ a bienes de capital, materia prima y bienes de consumo. Esta decisión fue provocada por la apreciación del dólar y la caída de precios del petróleo afectando a múltiples sectores siendo el sector automotriz uno de ellos, en referencia a su afectación económica, para el país e implantación en su sustentabilidad para ayudar al sector automotriz, en el Ecuador se divide en los cuatro grupos que son: 1. diversos económicos, 2. impactos ambientales, 3. impacto a los recursos o materiales, y 4. impactos sociales, es aquella que permite a las empresas entregar sus productos o servicios sin comprometer los recursos futuros o las habilidades de futuras generaciones.; en tal sentido en esta investigación analiza el impacto que han causado las salvaguardias en la situación económica del sector automotriz, mediante el análisis documental, financiero y económico de los ejercicios fiscales 2014 hasta el primer semestre del 2017.

Palabras claves: Salvaguardias, sostenibilidad, sector automotriz, rentabilidad, situación económica.

\section{Introducción.}

La salvaguardia o también llamada sobretasa arancelaria es una restricción temporal, por el aumento excesivo de las importaciones de un producto afectando significativamente a la economía de un país. Son como "medidas de urgencia" y son aplicables cuando causan un daño grave a la rama de producción de un país. Las salvaguardias "constituyen uno de los tres tipos de medidas especiales de protección del comercio internacional (los otros son las medidas antidumping y las medidas compensatorias) a las que pueden recurrir los Miembros de la OMC". (OMC, s/f); (Díaz, 2018)

Con Resolución 011-2015 emitida por el Comité de Comercio Exterior y puesta en vigencia el 11 de marzo de 2015 se estableció una sobretasa arancelaria de carácter temporal y no discriminatoria, con el propósito de regular el nivel general de importaciones y, de esta manera, salvaguardar el equilibrio de la balanza de pagos, conforme al porcentaje ad valorem determinado para las importaciones a consumo de las subpartidas descritas en el anexo descrita en el Artículo 1 de dicha resolución. Además, se indica que quedan excluidas de la aplicación las importaciones provenientes de países de menor desarrollo relativo que son miembros de la Asociación Latinoamericana de Integración (ALADI) en este caso: Bolivia y Paraguay. (Díaz, 2018)

\section{Países que optaron las medidas de las salvaguardias}

La salvaguardia posee un enfoque de proteccionismo, tal es así que países de diferentes partes del mundo han aplicado de diferente manera su función, entre estas son: 


\section{Colombia}

La Resolución No. 0185 del 5 de agosto de 2013, por parte del Ministerio de Comercio Industria y Turismo, "por la cual se ordena la apertura de una investigación de carácter administrativo, con el objeto de definir la imposición de una medida de salvaguardia general a las importaciones de barras de hierro o acero, sin alear (barras corrugadas) y alambrones de hierro o acero, sin alear (alambrones corrugados), originarias de Países Miembros de la Organización Mundial del Comercio (OMC)”, abrió un gran debate en el sector siderúrgico nacional, con impacto aún a escala global. México, España y Turquía, por mencionar algunos, han mostrado su inconformismo por esta acción. (El Tiempo, 2014; Díaz, 2018)

Las salvaguardias aplicadas al alambrón de acero han generado un sobrecosto de un $16 \%$ a insumos básicos de los sectores de construcción de vivienda y agropecuario. (Díaz, 2018)

El diario agrega que "el problema es que las medidas de protección fueron adoptadas por Colombia para amparar a Acerías Paz del Río y Diaco, empresas controladas, respectivamente, por las brasileñas Votorantim y Gerdau” (El Tiempo, 2013); (Díaz, 2018)

\section{Costa Rica}

En la página web (Barquero, 2015) ; ( Díaz Sánchez, 2018) informa que la salvaguardia al arroz fue establecida el 19 de febrero del 2015 con un aumento del $35 \%$ al $60 \%$.

A lo que Central América Data (2017) comunica que el costo se incrementó casi en un 200\% entre los años 2011 al 2015, y aun así el producto importado puede competir con el producto local. A pesar de elevar el arancel a un 60\%, sus principales exportadores Argentina y Uruguay no han tenido dificultad en ser competencia para Costa Rica, aun así, solicitaron una compensación por el daño que ha causado dicho arancel y en definitiva el gobierno de Costa Rica se vio en la obligación de retribuir a dichos países con una cuota arrocera, es decir, se acordó que pueda exportar a Costa Rica un total de 3786 toneladas y estas quedaran exentas de dicho arancel, y tendrían que pagar solo el 35\%. "Esto evidencia la clara falta de competitividad de la producción local de arroz, que aún con el subsidio del Estado, enfrenta dificultades para competir con el producto extranjero".

\section{Chile}

El Diario el Economista América (2013) publicó que La Comisión Nacional de Distorsiones llegaron a la resolución de aplicar una salvaguardia del 9,7\% -sin fecha de término- a la importación de maíz grano, proveniente principalmente de Argentina y Estados Unidos. Según cifras de Odepa-Servicio Nacional de Aduanas, las importaciones de maíz partido y de maíz entero, indica que las importaciones totales crecieron un $13 \%$ en 2012 y un $23 \%$ en 2011, dando como resultado una recuperación menor a la esperada. Además, en el año 2012 se registró una caída del 16\%, demostrando que la aplicación de esta medida no ha sido de ayuda al país. En cuanto a la producción nacional su crecimiento ha sido moderado, pero sostenido y con una participación estable durante los último tres años en torno al 59\% del consumo. (Díaz, 2018)

\section{Sostenibilidad}

La sostenibilidad de la sociedad que se vinculan a la esfera de los servicios en que se encuentran los destinados la de salud y el medioambiente, pero estos no constituyen objeto de estudio de las asignaturas 
que se imparten. En consideración a estos aspectos y como una vía para ampliar los conocimientos que adquieren los estudiantes durante el aprendizaje, enfocada al medio ambiente y la responsabilidad social de las empresas el nuevo orden económico se traduce finalmente en un nuevo orden político y cultural que debe ser acompañado por modificaciones fundamentales en la forma de gestionar las empresas, en donde se crean activos valiosos de mercado, de infraestructura y de capital intelectual, los cuales deberán ser gestionados para el logro de los objetivos estratégicos propuestos. (Calvo, 2010); (Cañizares \& Suárez, 2018)

Argumenta (Rodríguez \& Zambrano, 2017) que una empresa sostenible es aquella que permite a las demás entregar sus productos o servicios sin comprometer los recursos futuros o las habilidades de futuras generaciones. De esta manera, una empresa es sostenible en la medida que logre ser simultáneamente rentable, carbono neutro, tener una baja huella hídrica, asegurar la disponibilidad de materiales para el futuro y genere bienestar para sus clientes, empleados y sociedad en general. (Tabla 1).

Tabla 1. Principales drivers económicos, impactos sociales y ambientales en la cadena de suministro de la industria automotriz

\begin{tabular}{|c|c|}
\hline Categoría & Aspectos \\
\hline Drivers económicos & $\begin{array}{l}\text { - Costo de materiales y servicios } \\
\text { - Costo de producción } \\
\text { - Costo de adquisición de los clientes } \\
\text { - Costo de final de ciclo de vida }\end{array}$ \\
\hline Impactos ambientales & $\begin{array}{l}\text { - Emisión de gases efecto invernadero } \\
\text { - Agotamiento del ozono estratosférico } \\
\text { - Creación de ozono fotoquímico } \\
\text { - Potencial de acidificación terrestre y marina } \\
\text { - Toxicidad ambiental y humana } \\
\text { - Formación de particulas peligrosas }\end{array}$ \\
\hline $\begin{array}{l}\text { Impacto en la disponibilidad } \\
\text { de recursos o materiales }\end{array}$ & $\begin{array}{l}\text { - Consumo de energía } \\
\text { - Consumo de agua } \\
\text { - Reciclaje y reutilización de materiales al final del } \\
\text { ciclo de vida } \\
\text { - Pérdida temporal de uso de suelo }\end{array}$ \\
\hline Impactos sociales & $\begin{array}{l}\text { - Seguridad del conductor y peatones } \\
\text { - Ruido de conducción } \\
\text { - Impacto de vibraciones del motor en el conductor } \\
\text { - Efectos en salud humana por calidad de aire } \\
\text { externo } \\
\text { - Calidad del aire al interior del vehículo } \\
\text { - Posibilidad de uso de vehículo por personas de } \\
\text { tercera edad, discapacitados } \\
\text { - Seguridad y salud ocupacional de empleados } \\
\text { - Derechos laborales y humanos }\end{array}$ \\
\hline
\end{tabular}

Fuente: (Rodríguez \& Zambrano, 2017)

\section{La sostenibilidad de la industria automotriz en el contexto de Ecuador}

Siendo uno de los principales sectores a nivel nacional en tecnología y potencial para innovar la matriz productiva, el sector automotriz tiene una importante participación a nivel nacional, así como también sus ventas, los principales productos que se importaron en el año 2016 informa el Servicio Nacional de Aduana 
del Ecuador (SENAE) fueron medicamentos, formas primarias y en tercer lugar los automóviles, considerando que se basan en el Costo, seguro y flete (CIF) (Tabla 2) (Díaz, 2018)

Tabla 2. Ventas anuales de automóviles en Ecuador

\begin{tabular}{lll}
\hline PRODUCTOS & CIF millones & \% Partic. CIF \\
\hline Medicamentos & 780 & $5,70 \%$ \\
Polímeros & 588 & $4,29 \%$ \\
Automóviles & 304 & $2,22 \%$ \\
$\begin{array}{l}\text { Residuos industria } \\
\text { alimentaria }\end{array}$ & 375 & $2,74 \%$ \\
Maquinarias y sus partes & 428 & $3,13 \%$ \\
Fundición de Hierro & 366 & $2,67 \%$ \\
Manufactura de plástico & 323 & $2,36 \%$
\end{tabular}

Fuente: (Díaz, 2018)

Tungurahua en los años 2015 y 2016 atravesaron por serios problemas para vender autos teniendo una disminución de hasta el $60 \%$, provocando una reducción de personal y eliminación algunos servicios que ofrecían, explican Javier Holguín, jefe de ventas de Automotores Carlos Larrea, la venta de autos usados en la ciudad para el año 2017 se ha incrementado la demanda hasta en un 50\%. Diego Salguero, gerente propietario de Automotores Salguero Sandoval, agrega que posee una mejora notoria desde el mes de abril, un motivo es la eliminación de impuestos lo que afectó a autos nuevos como a usados. El incremento de los cinco últimos meses fue del 15 al 20\%, informó el Diario (La Hora, 2017); (Díaz, 2018)

Las importaciones son el ingreso de mercadería foránea al territorio nacional. Se analiza que la lista al componente producto: 87 . Vehículos automóviles, tractores, velocípedos y demás vehículos terrestres, sus partes y accesorios corresponde : (Tabla 3.) (Díaz, 2018)

Tabla 3. Importaciones Sector Automotriz 2014 - 2017

\begin{tabular}{lllll}
\hline Importador & $\mathbf{2 0 1 4}$ (millones) & $\mathbf{2 0 1 5}$ (millones) & $\mathbf{2 0 1 6}$ (millones) & $\mathbf{2 0 1 7}$ (millones) \\
\hline Ecuador & $\$ 2.175$ & $\$ 1.536,7$ & $\$ 1.140,8$ & $\$ 1.878,5$ \\
\hline
\end{tabular}

Fuente: Díaz, P (2018)

Como se puede observar existió una disminución entre los años 2014 al 2015 de $-\$ 638$ millones equivaliendo a un $-41,54 \%$, en los años 2015 al 2016 con una variación de -\$395 millones dando como resultado un -34,70\%, y terminando en los años 2016 al 2017 aumentó en $\$ 737$ millones equivaliendo a un $39,27 \%$ 
Las importaciones se rigen a impuestos generales, obligaciones en materia de regulaciones y restricciones no arancelarias. (Leyva, 2004). Los tributos son la principal fuente de ingresos que ayuda al desarrollo socioeconómico y material de un país. (Tonato, 2017)

El catedrático "Pablo Rodríguez" en la obra "Tributación: historia y fundamentos" explica que: "el impuesto puede clasificarse en impuestos directos e indirectos: el impuesto directo es aquel que grava las fuentes de capacidad económica tales como rentas o patrimonios y el impuesto indirecto, es aquel que afecta al consumo y al gasto".

En el diario Expreso (Lisbeth, 2018) informa que al término de las salvaguardias hubo un aumento en las importaciones y por ende un crecimiento en las tasas indirectas en un 30\%, reportando el IVA un crecimiento del 8,2\%, pero se destaca que no se debe al crecimiento del sector interno. Además, que solo hasta febrero de este año, según cifras del Banco Central, las compras crecieron un $22 \%$. Para el sector comercial el efecto sin salvaguardias es positivo y se destaca también que al existir un crecimiento en la importación y tributos el sector se convierte dependiente a las importaciones, igualmente al traer mercadería extranjera se crea un stock que se vuelve a comprar después de un tiempo determinado afectando (Tabla 4).

Tabla 4. Recaudaciones IVA en importaciones $2014-2017$

\begin{tabular}{llllll}
\hline TIPO & $\begin{array}{l}\text { Impuesto al valor } \\
\text { Agregado }\end{array}$ & $\begin{array}{l}\text { Recaudación } \\
\text { Ene-Dic 2014 }\end{array}$ & $\begin{array}{l}\text { Recaudación } \\
\text { Ene-Dic 2015 }\end{array}$ & $\begin{array}{l}\text { Recaudación } \\
\text { Ene-Dic 2016 }\end{array}$ & $\begin{array}{l}\text { Recaudación } \\
\text { Ene-Dic 2017 }\end{array}$ \\
\hline Indirectos & IVA Importaciones & 2.035 .045 & 1.722 .178 & 1.329 .296 & 1.645 .546 \\
\hline
\end{tabular}

Fuente: Extraído a partir del SRI (2018)

Como se observa en los años 2014 al 2015 existió una disminución de $-\$ 312.867,40$ equivaliendo a una variación del $-18,17 \%$, de los años 2015 al 2016 una disminución de $-\$ 392.881,49$ dando una variación del $-29,56 \%$, y, por último, en los años 2016 al 2017 un aumento de $\$ 316.249,89$ equivaliendo a un 19,22\%. Adicionalmente, en los años 2014 y 2017 existió una disminución de \$389.498,99.

En consecuencia, se analizará el impacto causado las salvaguardias en la situación económica del sector automotriz en los períodos del 2014 al primer semestre del 2017.

\section{Metodología.}

Dentro de la lógica de intervención se aplicó la revisión bibliográfica y documental basada específicamente en la toma de libros, base de datos de páginas web, revistas, periódicos, estadísticas, archivos, folletos, sistemas de información computarizados, dentro de lo más relevante se han considerado los datos extraídos de las páginas web de la Superintendencia de Compañías y Banco Central del Ecuador. El enfoque con mayor trascendencia será el cuantitativo, debido a que la información recolectada es a partir de los Estados de Resultados de las empresas del sector automotriz y los productos que han sido afectados por la aplicación de la medida serán analizadas por base de datos de los organismos pertinentes. 
Los estudios de alcance descriptivos especifican las propiedades, características, perfiles o procesos o cualquier situación objeto de estudio como lo dice su autor (Hernández, 2014), la problemática que se presenta en este proyecto de investigación se relaciona a nivel nacional, pero como objetos de estudio, se analizará al sector de la provincia de Tungurahua.

\section{Población}

Mediante la "Clasificación Nacional de Actividades Económicas CIIU 4", el cual "clasifica uniformemente las actividades o unidades económicas de producción, dentro de un sector de la economía, según la actividad económica principal" (INEC, 2012);(Díaz, 2018), en donde explica que es una estructura de clasificación coherente y consistente de actividades económicas, basándose en conceptos, definiciones y normas, de esta manera da un marco general de los datos que puedan reunir y divulgarse dando oportunidad a su análisis económico. Para su clasificación se basan en insumos de bienes, servicios y factores de producción, proceso y la tecnología de producción, características, categorías de cada nivel de la clasificación son mutuamente excluyentes

La población serán las empresas inscritas en la Superintendencia de Compañías que se identifica por medio de la "Clasificación Nacional de Actividades Económicas CIIU 4" los cuales se subdividen en componentes: G45 comercio y reparación de vehículos automotores y motocicletas, C29 fabricación de vehículos automotores, remolques y semirremolques y C22 fabricación de productos de caucho y plástico, cuyos datos se detallan a continuación, el resultado fue que 92 empresas pertenecientes al sector automotriz o realizan actividades relacionadas al sector en la provincia de Tungurahua (Tabla 5). (Díaz, 2018)

Tabla 5. Clasificación Nacional de Actividades Económicas CIIU 4

\begin{tabular}{|c|c|}
\hline $\begin{array}{l}\text { Código } \\
\text { Clasifica- } \\
\text { dor }\end{array}$ & Descripción \\
\hline G45 & Comercio y Reparación De Vehículos Automotores Y Motocicletas. \\
\hline G451 & Venta de Vehículos Automotores. \\
\hline G4510 & Venta de Vehículos Automotores. \\
\hline G4510.0 & Venta de Automóviles y Vehículos de Motores Ligeros. \\
\hline G4510.01 & $\begin{array}{l}\text { Venta de vehículos nuevos y usados: vehículos de pasajeros, incluidos vehículos especializados como: } \\
\text { ambulancias y minibuses, camiones, remolques y semirremolques, vehículos de acampada como: caravanas } \\
\text { y auto caravanas, vehículos para todo terreno (jeeps, etcétera), incluido la venta al por mayor y al por menor } \\
\text { por comisionistas. }\end{array}$ \\
\hline G452 & Mantenimiento y reparación de vehículos automotores. \\
\hline G4520 & Mantenimiento y reparación de vehículos automotores. \\
\hline G4520.0 & Mantenimiento y reparación de vehículos automotores. \\
\hline G4520.01 & $\begin{array}{l}\text { Mantenimiento y reparación de vehículos automotores: reparación mecánica, eléctrica, sistemas de inyec- } \\
\text { ción eléctricos, carrocerías, partes de vehículos automotores: parabrisas, ventanas, asientos y tapicerías. } \\
\text { Incluye el tratamiento anti óxido, pinturas a pistola o brocha a los vehículos y automotores, la instalación } \\
\text { de partes, piezas y accesorios que no se realiza como parte del proceso de fabricación (parlantes, radios, } \\
\text { alarmas, etcétera). }\end{array}$ \\
\hline G453 & Venta de partes, piezas y accesorios para vehículos automotores. \\
\hline G4530 & venta de partes, piezas y accesorios para vehículos automotores. \\
\hline G4530.0 & Venta de partes, piezas y accesorios para vehículos automotores. \\
\hline $\mathrm{C} 29$ & Fabricación de vehículos automotores, remolques y semirremolques. \\
\hline
\end{tabular}


C291 Fabricación de vehículos automotores.

C2910 Fabricación de vehículos automotores.

C2910.0 Fabricación de vehículos automotores.

C2910.01 Fabricación de automóviles de pasajeros, vehículos para todo terreno, autobuses, trolebuses, go-carts y vehículos similares, incluidos vehículos de carreras.

C2910.02 Fabricación de vehículos para el transporte de mercancías: camionetas, camiones, tractores para semirremolques de circulación por carretera, etcétera.

C2910.03 Fabricación de motores para vehículos automotores.

C2910.04 Fabricación de chasis equipados con motores.

Fabricación de otros vehículos automotores: trineos motorizados, carritos autopropulsados para campos de

C2910.05 golf, vehículos anfibios, camiones de bomberos, camiones barredores, bibliotecas móviles, vehículos blindados, camiones hormigonera, etcétera.

C2910.06 Servicios de apoyo a la fabricación de vehículos automotores, reconstrucción, rectificación en fábrica de sus motores a cambio de una retribución o por contrato.

C292 Fabricación de carrocerías para vehículos automotores; fabricación de remolques y semirremolques.

C2920

C2920.0

Fabricación de carrocerías para vehículos automotores; fabricación de remolques y semirremolques.

C2920.01

Fabricación de carrocerías para vehículos automotores; fabricación de remolques y semirremolques.

C2920.02

C2920.03

C2920.04

C293

C2930

Fabricación de carrocerías, incluidas cabinas para vehículos automotores.

Fabricación de remolques y semirremolques: para el transporte de mercancías: camiones cisterna, de mudanzas, etcétera, para el transporte de pasajeros: caravanas, etcétera.

C2930.0

Fabricación de contenedores para su acarreo por uno o más medios de transporte.

Servicios de apoyo a la fabricación, ensamblaje y equipamiento de carrocerías para vehículos automotores, remolques y semirremolques a cambio de una retribución o por contrato.

C2930.01 Fabricación de partes, piezas y accesorios de carrocerías para vehículos automotores: cinturones de seguridad, dispositivos inflables de seguridad (airbag), puertas, parachoques, asientos.

Fabricación de equipo eléctrico para vehículos automotores, como generadores, alternadores, bujías, ca-

C2930.02 bleados preformados para el sistema de encendido, sistemas eléctricos de apertura y cierre de ventanillas y puertas, montaje de tableros de instrumentos, reguladores de tensión, etcétera.

C2930.03

Servicios de apoyo a la fabricación de partes, piezas y accesorios para vehículos automotores a cambio de una retribución o por contrato.

Fabricación de otras partes, piezas y accesorios para vehículos automotores: frenos, cajas de cambios, ejes,

C2930.09 aros de ruedas, amortiguadores, radiadores, silenciadores, tubos de escape, catalizadores, embragues, volantes, columnas y cajas de dirección, etcétera.

C22 Fabricación de productos de caucho y plástico.

C221 Fabricación de productos de caucho.

C2211 Fabricación de cubiertas y cámaras de caucho; recauchutado y renovación de cubiertas de caucho.

C2211.0 Fabricación de cubiertas y cámaras de caucho; recauchutado y renovación de cubiertas de caucho.

C2211.01 Fabricación de cubiertas de caucho para: vehículos, equipo, maquinaria móvil, aeronaves, juguetes, muebles y otros usos: neumáticos, llantas.

fabricación de bandas de rodadura intercambiables, cámaras para cubiertas, fajas de protección de la cá-

C2211.02 mara, tiras de remiendo para recauchutar cubiertas, etcétera, renovación y recauchutado de cubiertas de neumáticos sean cubiertas sólidas y mullidas (esponjosas, blandas). 
Elaboración propia a partir de la Superintendencia de compañías (2018)

\section{Muestra}

De acuerdo a la metodología de la investigación y aplicando el muestreo $(\mathrm{z}=1,96$ y e $=5 \%)$ correspondería a un mínimo de 75 empresas, pero se usó el muestreo no probabilístico intencional en función a que solo 60 empresas aplica con los requisitos necesarios para esta investigación, es decir, poseen los estados financieros de los años 2014 al 2017 brindando los datos requeridos para el análisis respectivo, el resto de empresas se excluyen por no cumplir con las exigencias de información disponible en consecuencia en este estudio se trabajará con un número de sesenta empresas. (Díaz, 2018)

Unidad de análisis

Partiendo de la Clasificación Nacional de Actividades Económicas CIIU 4 que permite obtener una población más estratificada la siguiente lista de empresas encajan con los requisitos para su estudio (Tabla 6). (Díaz, 2018)

Tabla 6. Unidad de análisis

\begin{tabular}{|c|c|}
\hline $\mathbf{N}^{\circ}$ & EMPRESA \\
\hline 1 & Pico Sánchez Cía. Ltda. \\
\hline 2 & Industria Nacional De Retrovisores Y Cromados S.A. INARECROM \\
\hline 3 & Varma S.A. \\
\hline 4 & Davmotor Cía. Ltda. \\
\hline 5 & Patricio Cepeda Cía. Ltda. \\
\hline 6 & Esprom-Pur Cía. Ltda. \\
\hline 7 & Automotores De La Sierra SA. \\
\hline 8 & Ambacar Cía. Ltda. \\
\hline 9 & Importadora Alvarado Vascones C Ltda. \\
\hline 10 & Marielhez Cía. Ltda. \\
\hline 11 & Pareco Cía. Ltda. \\
\hline 12 & Ladias Laboratorios Diésel Ambato S.A. \\
\hline 13 & Ambandine S.A. \\
\hline 14 & Autos Y Servicios De La Sierra Autosierra S.A. \\
\hline 15 & Automotores Carlos Palacios E Hijos Cía. Ltda. \\
\hline 16 & González Vega El Motor Cía. Ltda. \\
\hline 17 & Iavgroup Cía. Ltda. \\
\hline 18 & Automotores Del Pacifico S.A. Autollegasa \\
\hline 19 & Vihalmotos Importaciones S.A. \\
\hline 20 & Talleres Ambamazda S.A. \\
\hline 21 & Automotores Carlos Larrea T. Cía. Ltda. \\
\hline 22 & Comercial Cisneros Importadora Kumho Cía. Ltda. \\
\hline 23 & Toyotaservicios S. A. \\
\hline 24 & Llantas Y Servicios De La Sierra, Llantasierra S.A. \\
\hline 25 & Automekano Cía. Ltda. \\
\hline
\end{tabular}




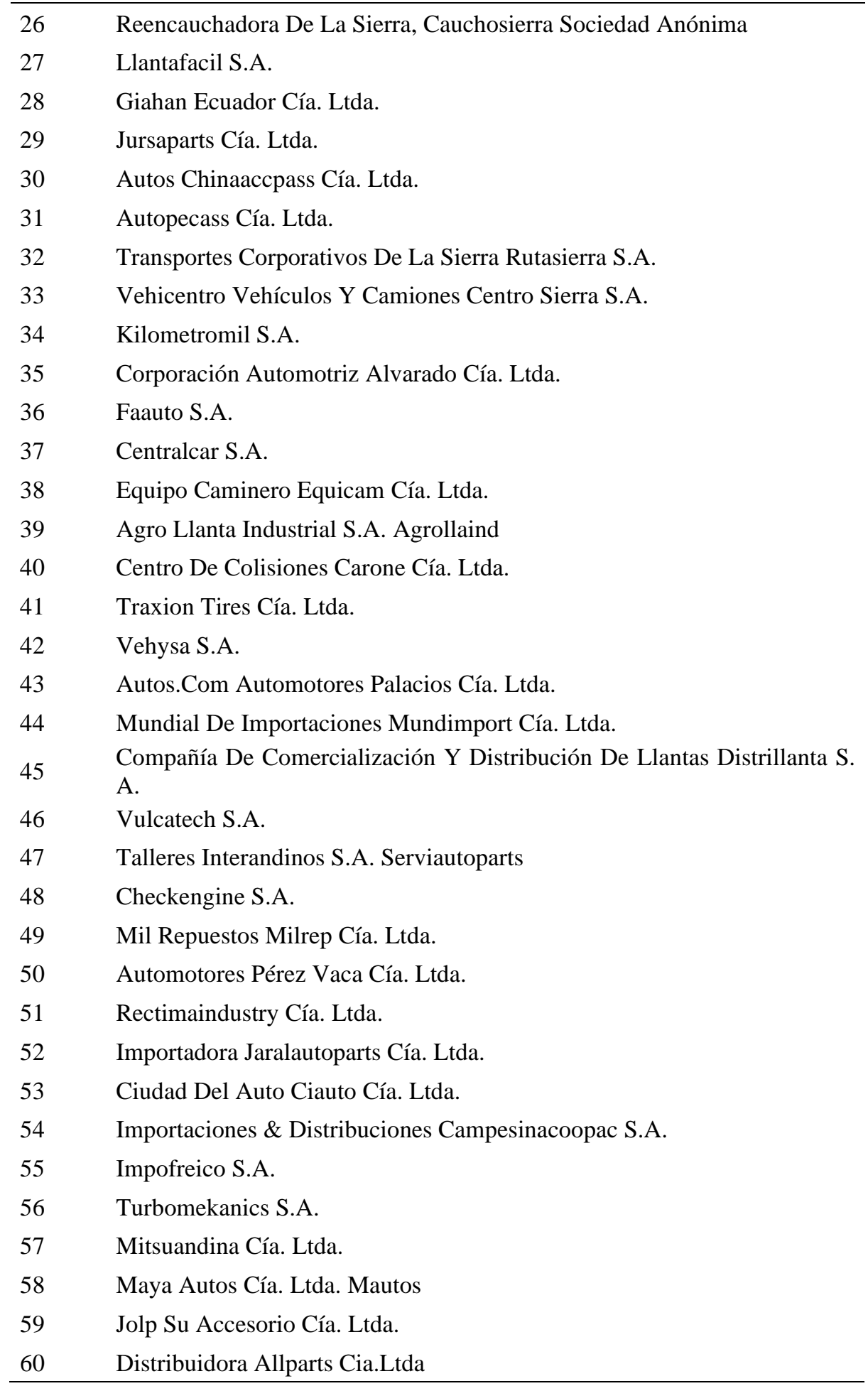

Elaboración propia a partir de la Superintendencia de compañías (2018)

Plan de recolección de la información 
El plan de recolección de la información consiste en la reunión de herramientas y técnicas ordenadas que utilizó el investigador para la obtención de los datos requeridos para su investigación (Zapata \& Rivera, 2011); (Díaz, 2018). Con este argumento se refiere a la búsqueda de la población y muestra. Como primera fase la población se localizó por medio de Clasificación Nacional de Actividades Económicas CIIU descargada de la página de la Superintendencia de Compañías dando como resultado las empresas a analizar aplicando las técnicas de observación, computación y revisión selectiva. Así, como también un análisis externo puesto que solo se maneja la información de la Superintendencia de Compañías se podrá recopilar, interpretar y comparar las cuentas contables en los estados.

Plan de procesamiento de la información

Por medio de los estados financieros desde el 2014 al 2017 y con análisis horizontales entre los años 2014 al 2015, 2015 al 2016 y 2016 al 2017 se calculará las variaciones relativas y diferencias de esta manera se estudiará las subcuentas que lo componen y el estudio de cada uno de ellos. Por medio de los indicadores de rentabilidad se interpretó los resultados de como controlaron las empresas los costos y gastos para generar utilidad, de esta manera se evaluó el desempeño financiero y operacional de cada empresa que se aplicó en la muestra, lo que ayudó al investigador a comprender por medio de un enfoque amplio y detallado la naturaleza del sector y sus limitaciones a través de estos años y se fijó si las políticas empresariales y gubernamentales cumplieron con el objetivo de mejorar el sector e incentivar el producto nacional.

\section{Resultados.}

El análisis de las cuentas contables que representadas en los estados financieros de los ejercicios fiscales terminados al 31 de diciembre de 2014 al 2017, reflejan que el Activo subió en \$15'885.440,38, de igual manera las Ventas con un aumento de $\$ 51^{\prime} 693.458,91$, continuando con el costo de ventas un incremento de $\$ 36$ '571.954,68, sin embargo, con la cuenta Utilidad Neta tiene una disminución de $\$ 553.524,81$ y por último con el patrimonio un aumento de $\$ 22 ’ 925.701,21$. Con esto se puede señalar que todas las cuentas analizadas tienen un aumento al 2017 sin embargo, la utilidad de las empresas disminuyó afectando negativamente al sector.

En tal sentido, se prueba que uno de los objetivos de las salvaguardias no se cumplió en este sector, a pesar de que subió en gran proporción las cuentas del 2014 al 2017, la utilidad que es el beneficio principal de una empresa y razón importante de la creación de una, se obtuvo un efecto negativo de 553.524,81. (Tabla 7.); (Figura 1.)

Tabla 7. Análisis Cuentas Contables

\begin{tabular}{lrrrrrr}
\hline AÑOS & Activo & \multicolumn{2}{l}{ Ventas } & Costo de Ventas Utilidad Bruta & Utilidad Neta & Patrimonio \\
\hline 2014 & $55^{\prime} 610.129,45$ & $41^{\prime} 802.851,13$ & $25^{\prime} 842.898,33$ & $15^{\prime} 959.952,80$ & $2{ }^{\prime} 501.297,17$ & $10^{\prime} 692.715,99$ \\
2015 & $54^{\prime} 970.123,52$ & $58^{\prime} 501.863,61$ & $45^{\prime} 903.965,84$ & $12^{\prime} 597.897,77$ & $954.897,25$ & $14^{\prime} 232.119,67$ \\
2016 & $49^{\prime} 044.308,72$ & $57^{\prime} 047.785,94$ & $41^{\prime} 849.377,02$ & $15^{\prime} 198.408,92$ & $929.039,33$ & $19^{\prime} 216.335,82$ \\
2017 & $71^{\prime} 495.569,83$ & $93^{\prime} 496.310,04$ & $62^{\prime} 414.853,01$ & $31^{\prime} 081.457,03$ & $1^{\prime} 947.772,35$ & $33^{\prime} 618.417,20$
\end{tabular}

Elaboración propia a partir de la Superintendencia de Compañías (2018) 
Figura 1. Análisis cuentas contables

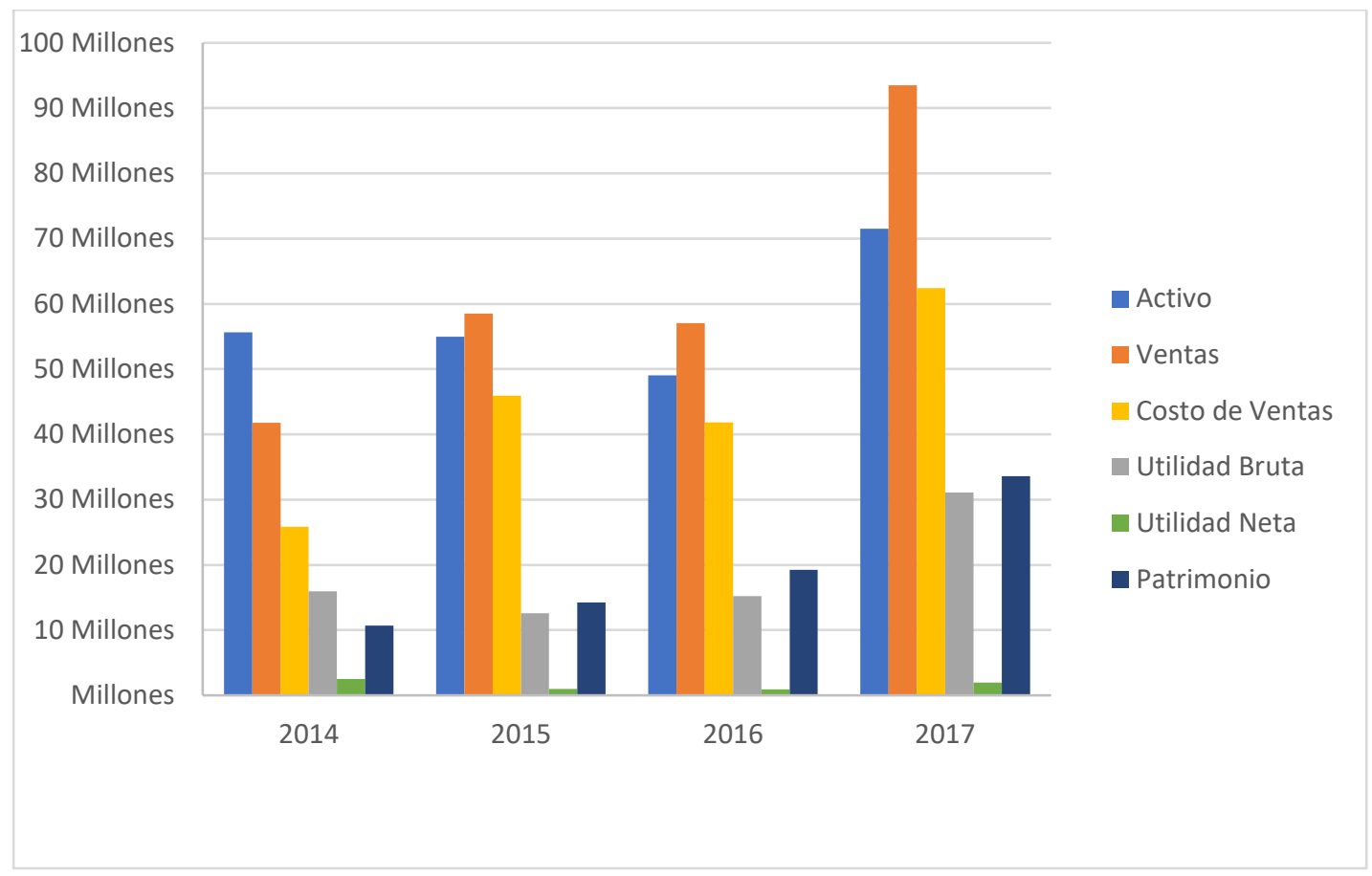

Elaboración propia a partir de la Superintendencia de Compañías (2018)

\section{Indicadores de Rentabilidad}

Se puede indicar que en los años del 2014 al 2017 el indicador rentabilidad neta del activo se redujo en $1,78 \%$, de igual manera el margen bruto disminuyó en un 7,44\%, sin embargo, el margen operacional subió en un 3.34\%, siguiendo con la rentabilidad neta de ventas con una disminución del 3,90\% y por último la rentabilidad operacional del patrimonio con un descenso significativo de 43,32\%. Con esto se puede señalar que la mayoría de indicadores del año 2014 respecto al 2017 sufrieron una caída y se vieron afectados significativamente, algo que se resalta fue el aumento del margen operacional lo que revela es que los ingresos provienen directamente de la actividad económica del sector. (Tabla 8); (Figura 2)

Tabla 8. Análisis Indicadores de Rentabilidad

\begin{tabular}{cccccc}
\hline AÑOS & $\begin{array}{c}\text { Rentabilidad Neta Margen Bruto Margen Operacional } \\
\text { del Activo }\end{array}$ & $\begin{array}{c}\text { Rentabilidad } \\
\text { Neta de Ventas }\end{array}$ & $\begin{array}{c}\text { Rentabilidad Operacional } \\
\text { del Patrimonio }\end{array}$ \\
\hline 2014 & $4,50 \%$ & $37,47 \%$ & $16,04 \%$ & $5,98 \%$ & $62,71 \%$ \\
2015 & $1,74 \%$ & $21,04 \%$ & $2,62 \%$ & $1,63 \%$ & $10,77 \%$ \\
2016 & $1,89 \%$ & $25,92 \%$ & $11,21 \%$ & $1,63 \%$ & $33,28 \%$ \\
2017 & $2,72 \%$ & $30,03 \%$ & $19,38 \%$ & $2,08 \%$ & $19,38 \%$ \\
\hline
\end{tabular}

Elaboración propia a partir de la Superintendencia de Compañías (2018) 
Figura 2. Análisis Indicadores de Rentabilidad

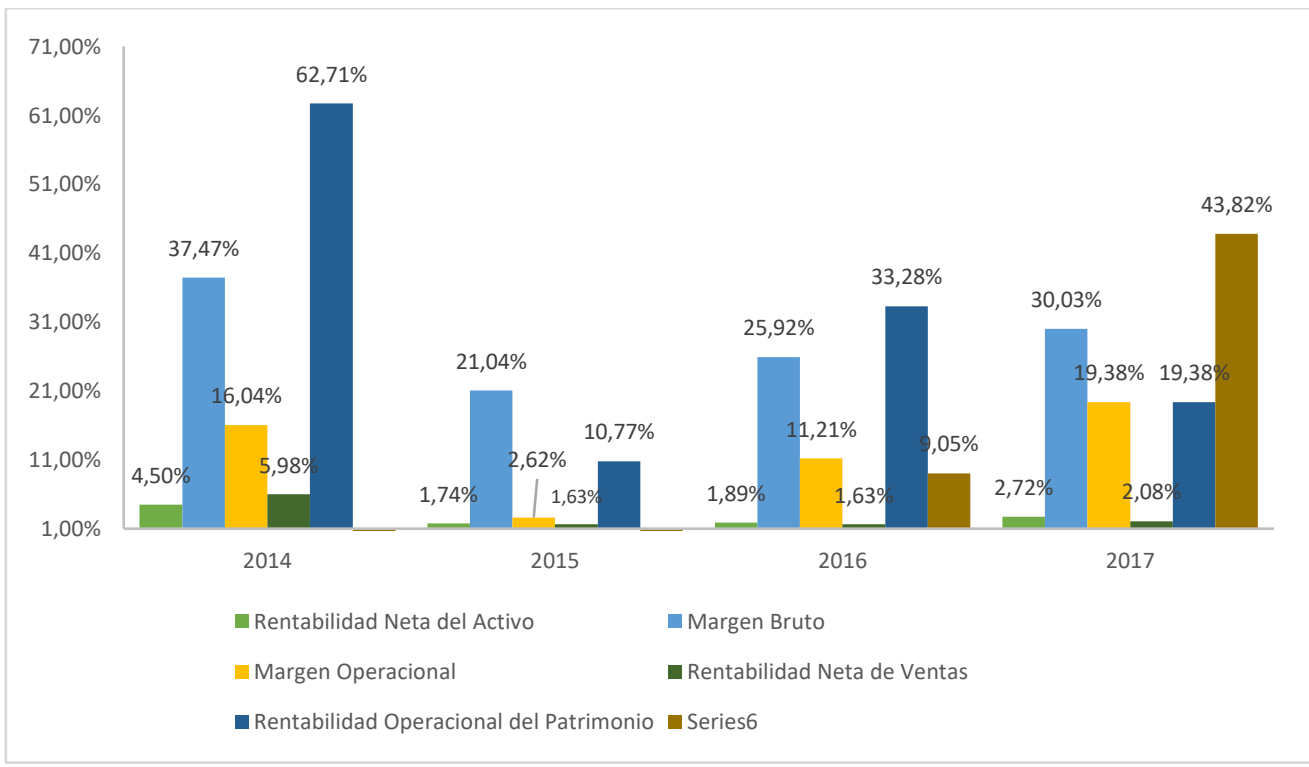

Elaboración propia a partir de la Superintendencia de Compañías (2018)

\section{Empresas que se declararon con pérdidas}

La muestra es de sesenta empresas a estudiar, sin embargo, es primordial destacar el número de empresas que declararon con pérdidas en los años del 2014 al 2017 donde se señala que 9 empresas en el año 2014 tuvieron pérdida equivaliendo a un 17,31\%, en el año 2015 fueron afectadas 10 empresas representando en un 19,23\%, en el año 2016 que destaca con 20 empresas equivaliendo al 38,46\% finalizando en el año 2017 con 13 empresas representando un $25 \%$.

El año 2016 fue el más afectado en cuanto a número de empresas en pérdida debido a que a las salvaguardias comenzaron con su paulatino desmantelamiento en junio de 2016 en su primera etapa y en octubre de 2016 su segunda, es decir, que la presencia de salvaguardias afectó significativamente en los años 2016 y 2015 llegando al punto de aumentar el número de empresas con bajo rendimiento. (tabla 9.); (figura 3.)

Tabla 9. Empresas con Pérdidas

\begin{tabular}{lll}
\hline AÑOS & Número de empresas con pérdidas & PORCENTAJE \\
\hline 2014 & 9 & $17,31 \%$ \\
2015 & 10 & $19,23 \%$ \\
2016 & 20 & $38,46 \%$ \\
2017 & 13 & $25,00 \%$ \\
TOTAL & $\mathbf{5 2}$ & $100 \%$
\end{tabular}

Elaboración propia a partir de la Superintendencia de Compañías (2018) 
Figura 3. Empresas con Pérdidas

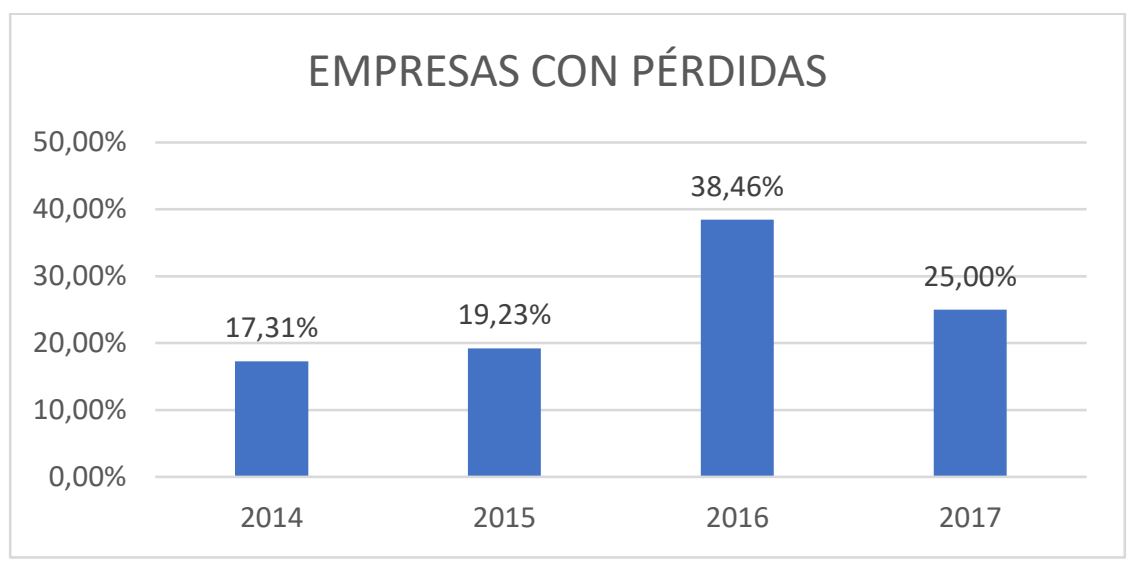

Elaboración propia a partir de la Superintendencia de Compañías (2018)

\section{Ventas sobre Importaciones}

Partiendo de la cuenta importaciones muestra la influencia de las salvaguardias en la provincia siendo en los años 2014 al 2015 con una disminución de \$-16’753.012,85 equivaliendo a un 14,36\%, en los años 2015 al 2016 disminuyo nuevamente con $\$-14$ '145.992,73 siendo un $14,16 \%$, pero destacando su incremento en los 2016 al 2017 con \$ 91'375.572,56 equivaliendo a un 106,54\%; además se observa que en el año 2014 al 2017 hubo un aumento de \$60`476.566,98 indicando que la presencia de las salvaguardias afecta que en su desaparición sea más marcada. (tabla 10.); (figura 4.)

Tabla 10. Importaciones

\begin{tabular}{llrlll}
\hline Años & \multicolumn{2}{l}{ Importaciones } & Variación Absoluta & Variación Relativa \\
\hline $\mathbf{2 0 1 4}$ & $\$$ & $116.662 .735,42$ & & & \\
$\mathbf{2 0 1 5}$ & $\$$ & $99.909 .722,57$ & $\$$ & $-16.753 .012,85$ & $-14,36 \%$ \\
$\mathbf{2 0 1 6}$ & $\$$ & $85.763 .729,84$ & $\$$ & $-14.145 .992,73$ & $-14,16 \%$ \\
$\mathbf{2 0 1 7}$ & $\$$ & $177.139 .302,40$ & $\$$ & $91.375 .572,56$ & $106,54 \%$ \\
\hline
\end{tabular}

Elaboración propia a partir de la Superintendencia de Compañías (2018) 
Figura 4. Importaciones

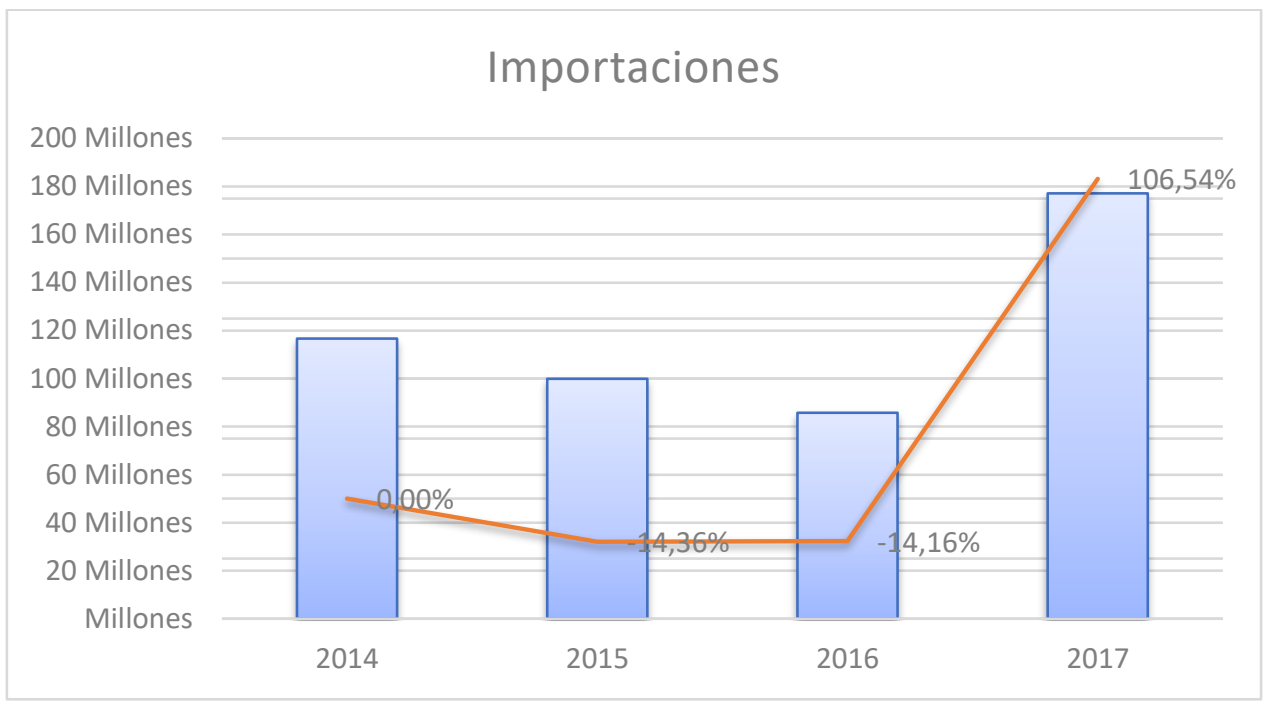

Elaboración propia a partir de la Superintendencia de Compañías (2018)

Importaciones sobre las Ventas.

En los años 2014 al 2015 existió una disminución en salvaguardias y se incrementaron las ventas, pero en los años 2015 al 2016 hubo una disminución en importaciones, así como también sus ventas y finalizando en el 2016 al 2017 hubo un aumento de importaciones destacable pero un aumento en ventas moderado. (tabla 11.); (figura 5.)

Tabla 11. Ventas sobre Importaciones

\begin{tabular}{ccccc}
\hline \multicolumn{1}{c}{ Años } & & Importaciones & & Ventas \\
\hline $\mathbf{2 0 1 4}$ & $\$$ & $116.662 .735,42$ & $\$$ & $41.802 .851,13$ \\
$\mathbf{2 0 1 5}$ & $\$$ & $99.909 .722,57$ & $\$$ & $58.501 .863,61$ \\
$\mathbf{2 0 1 6}$ & $\$$ & $85.763 .729,84$ & $\$$ & $57.047 .785,94$ \\
$\mathbf{2 0 1 7}$ & $\$$ & $177.139 .302,40$ & $\$$ & $93.496 .310,04$ \\
\hline
\end{tabular}

Elaboración propia a partir de la Superintendencia de Compañías (2018) 
Figura 5. Importaciones sobre las Ventas

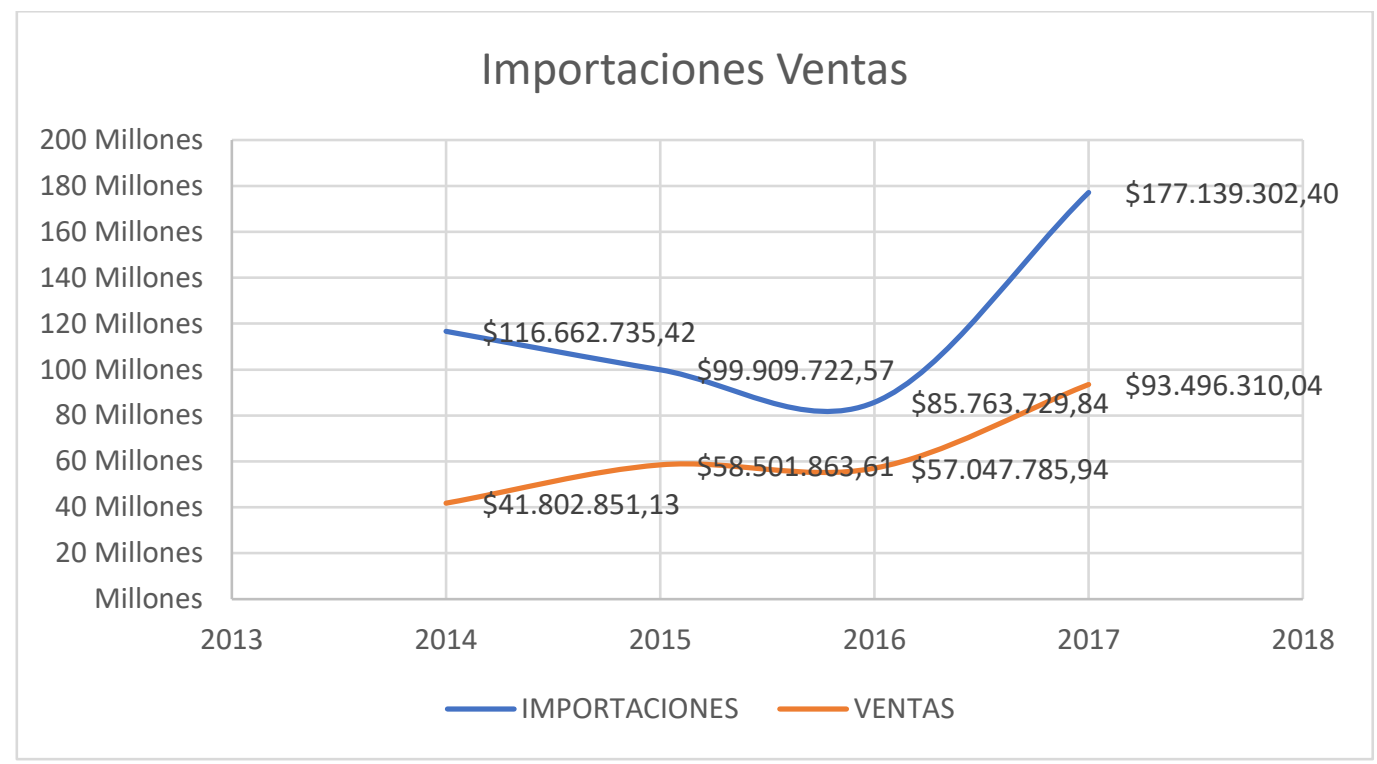

Elaboración propia a partir de la Superintendencia de Compañías (2018)

Como limitación de este estudio se resaltan las pequeñas empresas no obligadas a llevar contabilidad no se encuentran como objeto de estudio debido a que no tienen ingresos suficientes como para que requieran un control unificado obligatorio y así poder conocer su rendimiento real.

\section{Situación económica}

El sector automotriz tiene un impacto significativo en la economía de un país puesto que interviene en el transporte de personas y productos generando actividades comerciales en diferentes ámbitos: trabajo, pago de impuestos, aranceles, tasas e impuestos. Hay que tener en cuenta que el sector no solo se conforma por la comercialización de vehículos incluye también firmas autopartistas, ensambladoras, mantenimientos, reparación.

El boom de la producción automotriz en Ecuador empezó en la década de los años 50, cuando empresas del sector metalmecánico y del sector textil comenzaron la fabricación de carrocerías, asientos para buses, algunas partes y piezas metálicas, actualmente la contribución de la industria automotriz tiene un gran peso en el aparato económico nacional, se han ensamblado vehículos por más de tres décadas, en el año 1973 comenzó la fabricación de vehículos, con un total de 144 unidades de un solo modelo, conocido en aquel entonces como el Andino, ensamblado por AYMESA hasta el año 1980.

La presencia de empresas multinacionales en Ecuador, han liderado la transferencia y asimilación de tecnologías en empresas de autopartes y de ensamblaje de automóviles, lo cual se ve reflejado en el desarrollo tecnológico alcanzado por la industria automotriz ecuatoriana. De esta manera, la industria de ensamblaje ha brindado la oportunidad de la producción local de componentes, partes, piezas e insumos en general lo que genera a su vez un encadenamiento productivo en la fabricación de otros productos relacionados a los automotores, maquinarias y herramientas necesarias para producirlos". (Muñoz, 2016); (Díaz, 2018) (Figura 6). 
Figura 6. Conformación del Sector

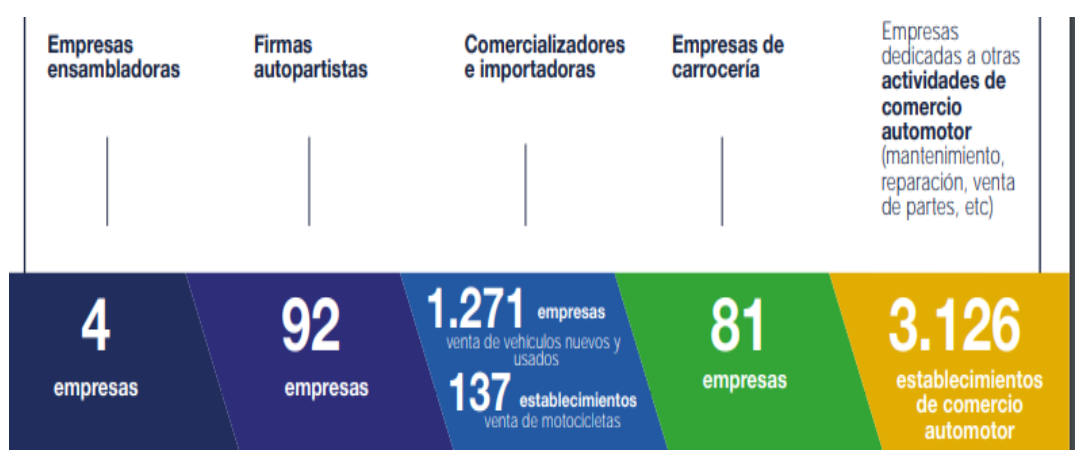

Fuente: Extraído a partir de la Superintendencia de Compañías (2016)

Las actividades relacionadas al sector automotriz generan un importante número de plazas de empleo. De acuerdo a información de la Superintendencia de Compañías (2016) se tienen 56.801 personas ocupadas, en los establecimientos de Comercio se encuentran ocupadas 35.994 personas (Figura 7).

\section{Figura 7. Generación de Puestos de Trabajo}

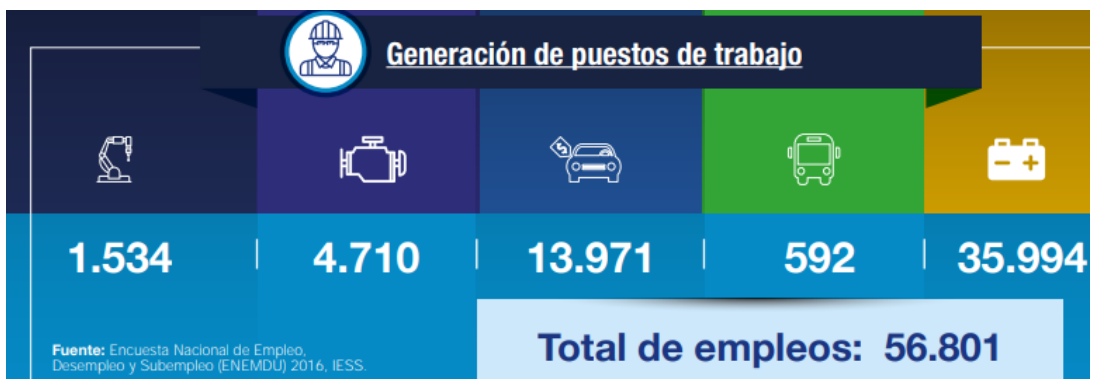

Fuente: Extraído a partir del IESS (2016)

En cuanto a las ventas de automóviles entre los años 2012 a 2015 del primer quimestre ha disminuido como se muestra en el siguiente gráfico con una diferencia de 17345 autos (Figura 8).

Figura 8. Cifras de comercialización

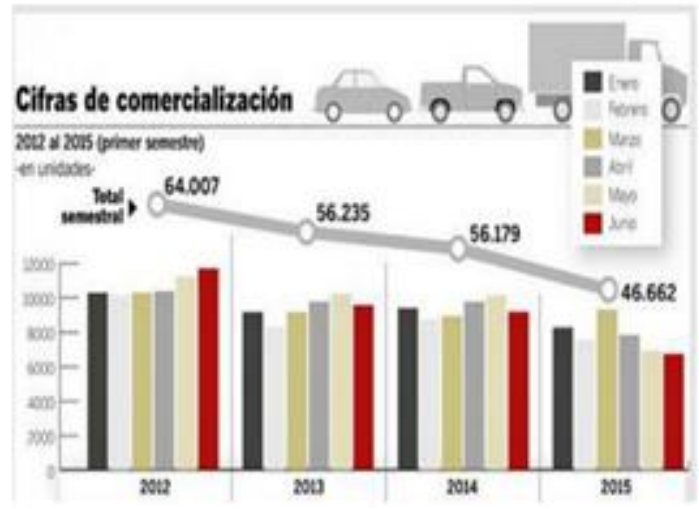

Fuente: Extraído a partir del diario El Universo (2015) 
El sector automotriz se ha visto afectado por varias medidas restrictivas, una de estas son la limitación de cupos a la importación de autos que tuvieron hasta el año 2016 comunico el Ministro de Comercio, Juan Carlos Cassinelli que a partir del 2017 se elimina esta medida debido a la crisis de este sector con una caída del 39\% en sus ventas (Sosa, 2016), hasta septiembre de 2016, el sector vendió 32692 unidades; lo que representó 20565 menos que igual período del año pasado, según datos de la Asociación de Empresas Automotrices del Ecuador (AEADE). En el 2015, el cupo para los autos importados era de USD 300 millones anuales, que se repartía entre 46 importadores. Para el 2016, según la Resolución 050 del Comité de Comercio Exterior (COMEX), el cupo bajó a USD 280 millones.

\section{Conclusiones.}

- La aplicación de las salvaguardias como medida para proteger el producto nacional que se afecta por el exceso de importaciones según el artículo XIX del Acuerdo General sobre Aranceles Aduaneros y Comercio, y en el artículo XVIII, sección B, por este hecho el Gobierno ecuatoriano se apoya para su implementación, pero no se midió ni realizó previas investigaciones a los sectores como lo han hecho otros países, considerando la solicitud de ciertas empresas para su apoyo y en este caso afectó significativamente al sector automotriz en el año 2015 y 2016 su utilidad obtuvo variaciones negativas del $-61,82 \%$ y $-2,71 \%$ respectivamente y con la ausencia de salvaguardias a partir de junio del 2017 obtuvo una alza del 109,65\% en su utilidad.

- Las resoluciones del Ministerio del Comercio Exterior en el Ecuador, se dividieron en tres fases el desmantelamiento de las salvaguardias a partir de junio 2016 y después su desmantelamiento paulatino hasta junio 2017, provocaron un aumento de capital en los años 2015, 2016 y 2017 equivaliendo al 33,10\%, 35,02\% y $74,95 \%$ respectivamente, es decir, que las empresas arriesgaron e introdujeron capital propio para sobrellevar esta medida y de esa manera estabilizar su costo de ventas.

- Las decisiones estratégicas de las empresas en la industria automotriz permiten la identificación de oportunidades de negocio y de riesgos, que pueden o no poner en peligro la continuidad de la empresa en el largo plazo.

\section{Referencias bibliográficas.}

Rodríguez, J., \& Zambrano, J. (2017). Industria Automotriz. ESPAE, 1-50. Recuperado el 13 de Diciembre de 2019, de http://www.espae.espol.edu.ec/wpcontent/uploads/2017/06/industriaautomotriz.pdf

Barquero, M. (14 de Abril de 2015). Costa Rica reduce arancel a cuota de arroz de Argentina y Uruguay. Obtenido de La Nación: https://www.nacion.com/economia/agro/costa-ricareduce-arancel-a-cuota-de-arroz-de-argentina-yuruguay/PC57TPRXTNFD5GCO3LMECLD53Y/story/

Calvo, J. (2010). Componente finanzas y su evolución. Apuntes Contables, 1-60. Recuperado el 14 de Diciembre de 2019, de https://revistas.uexternado.edu.co/index.php/contad/article/view/1663/1501

Cámara de la Industria Automotriz Ecuatoriana. (2018). CINAE presentó un detallado informe del sector y su "I Anuario de la Industria Automotriz Ecuatoriana". . Obtenido de https://www.cinae.org.ec/index.php?option=com_content \&view=article \&id=325:cinaepresento-detallado-informe-sector $\&$ catid=75\&Itemid $=193$ 
Cañizares, M., \& Suárez, A. (2018). Medioambiente y salud.pilares en asignaturas optativas de la carrera de contabilidad y finanzas.Cuba. Apuntes Contables, 1-14. Recuperado el 14 de Diciembre de 2019 , de https://revistas.uexternado.edu.co/index.php/contad/article/view/5389/6579

Díaz, P. (2018). Las salvaguardias y la incidencia económica del sector automotriz de la provincia de Tungurahua. 1-127. Recuperado el 20 de Diciembre de 2019, de https://repositorio.uta.edu.ec/bitstream/123456789/28551/1/T4334i.pdf

El Tiempo. (23 de Octubre de 2013). Queja contra Colombia ante la OMC por salvaguardias al acero. Obtenido de Redacción El Tiempo: http://www.eltiempo.com/archivo/documento/DR-106096

El Tiempo. (10 de Abril de 2014). Protegiendo al alambrón nacional. Obtenido de Redacción El Tiempo: http://www.eltiempo.com/archivo/documento/DR-866807

Hernández, R. (2014). Metodología de la Investigación. México : Mc Graw Hill.

INEC. (junio de 2012). Clasificación Nacional de Actividades Económicas CIIU 4.0. Obtenido de http://aplicaciones2.ecuadorencifras.gob.ec/SIN/metodologias/CIIU\%204.0.pdf

La Hora. (4 de Octubre de 2017). 80\% de crecimiento en venta de autos nuevos en Ambato. Obtenido de https://lahora.com.ec/tungurahua/noticia/1102104497/80_-de-crecimientoen-venta-de-autos-nuevos-en-ambato

La Hora. (20 de Septiembre de 2017). Crece la venta de autos usados en Ambato. Obtenido de https://www.lahora.com.ec/tungurahua/noticia/1102101289/crece-la-venta-de-autosusados-en-ambato

La Hora. (12 de enero de 2018). Al menos 10 años serían necesarios para bajar el peso de la deuda de Ecuador. Obtenido de https://lahora.com.ec/noticia/1102149188/al-menos-10anos-serian-necesarios-para-bajar-el-peso-de-la-deuda-de-ecuador

Leyva, E. (2004). Importaciones y Exportaciones ;Tratamiento Jurídico. México: ISEF empresa lider.

Lisbeth, Z. (25 de Abril de 2018). La importación eleva la recaudación de tributos. Obtenido de https://www.expreso.ec/economia/importaciones-economia-dinero-impuestos-ecuadorDB2146775

Ministerio de Industria y Productividad. (21 de junio de 2017). El diálogo con la industria automotriz se dinamiza. Obtenido de https://www.industrias.gob.ec/dialogo-con-industriaautomotriz-dinamiza/ 
Mochon, F., \& Carreon, V. (2014). Macroeconomía con apliaciones de América Latina. Santa Fe, México: Mc Graw Hill.

OMC. (s/f). Antidumping, subvenciones, salvaguardias: casos imprevistos, etc. Obtenido de https://www.wto.org/spanish/thewto_s/whatis_s/tif_s/agrm8_s.htm

Pro Ecuador. (2017). Análisis Sectorial Automotriz. Quito: Ministerio de Comercio Exterior.

Sosa, C. A. (30 de septiembre de 2016). Ecuador eliminará cupos a la importación de autos a partir del 2017 . Obtenido de http://www.elcomercio.com/actualidad/ecuador-eliminaracupos-importacion-autos.html

Tapia, E. (4 de Diciembre de 2017). Ecuador crecerá en el 2018, pero todavía depende del petróleo. Obtenido de http://www.revistalideres.ec/lideres/ecuador-crecera-2018-todaviadepende.html

Tonato, G. (2017). nálisis del arancel y de los impuestos, Valor Agregado (IVA), Consumos Especiales (ICE) y Salidas de Divisas (ISD), en las importaciones y su incidencia en la balanza comercial del país. Periodo 2005-2015. Quito: Universidad Andina Simón Bolívar.

Zapata, D., \& Rivera, J. (13 de Abril de 2011). Metodología de la investigación - Recolección de datos. Obtenido de https://prezi.com/a-luhdaspb9i/metodologia-de-la-investigacionrecoleccion-de-datos/

\section{Ciencia \\ Digital}


PARA CITAR EL ARTÍCULO INDEXADO.

Díaz Sánchez, P. K., Mayorga Díaz, M. P., López Jara, A. A. L. J., \& Espinosa Jaramillo, M. T. (2020). Las salvaguardias y la sostenibilidad del sector automotriz de la provincia de Tungurahua - año 2014 a junio del 2017. Visionario Digital, 4(2), 81-101. https://doi.org/10.33262/visionariodigital.v4i2.1216

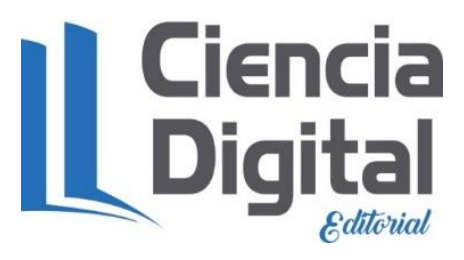

El artículo que se publica es de exclusiva responsabilidad de los autores y no necesariamente reflejan el pensamiento de la Revista Ciencia Digital.

El artículo queda en propiedad de la revista y, por tanto, su publicación parcial y/o total en otro medio tiene que ser autorizado por el director de la Revista Ciencia Digital.
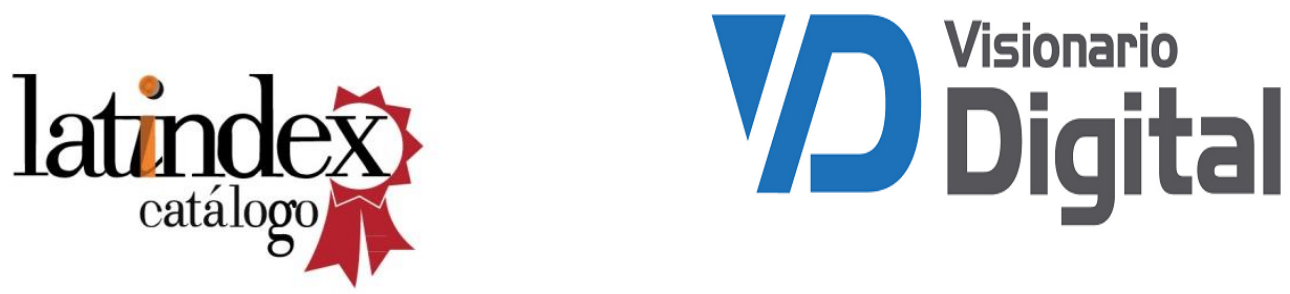